Gentao Cao - Xiaohua Ni • Min Jiang • Yushu Ma

Haipeng Cheng • Lingchen Guo • Chaoneng Ji

Shaohua Gu $\cdot$ Yi Xie · Yumin Mao

\title{
Molecular cloning and characterization of a novel human cAMP response element-binding (CREB) gene (CREB4)
}

Received: February 26, 2002 / Accepted: March 28, 2002

\begin{abstract}
Cyclic adenosine monophosphate (cAMP) response element-binding (CREB) proteins are a family of mammalian transcription activators. We identified a novel human $C R E B$ gene (CREB4) that was 1592 bp long and encoded a protein of 395 amino acid residues. The protein shared high homology to mouse CREB3 (identity $62 \%$, similarity $72 \%$ ). The expression pattern of the human CREB4 gene showed transcripts in prostate, brain, pancreas, skeletal muscle, small intestine, testis, leukocyte, and thymus, whereas in heart, lung, liver, kidney, placenta, spleen, ovary, and colon, specific bands of the transcript could not be detected. The CREB 4 gene consisted of ten exons and nine introns and was mapped to chromosome 1q21.3 by means of a bioinformatics analysis.
\end{abstract}

Key words CREB4 - MTC panel PCR - Chromosome 1q21.3 - Leucine zipper - Transcription activator

Extracellular stimuli elicit changes in gene expression in target cells by activating intracellular protein kinase cascades that phosphorylate transcription factors within the nucleus. The cyclic adenosine monophosphate (cAMP) response element-binding (CREB) protein, a stimulusinduced transcription factor, activates transcription of target genes in response to a diverse array of stimuli, including peptide hormones, growth factors, and neuronal activity. Such stimuli activate a variety of protein kinases, including protein kinase A (PKA), mitogen-activated protein kinases (MAPKs), and calmodulin-dependent protein kinases

G. Cao $\cdot$ X. Ni $\cdot$ M. Jiang $\cdot$ Y. Ma $\cdot$ H. Cheng $\cdot$ L. Guo $\cdot$ C. Ji

S. Gu $\cdot$ Y. Xie $\cdot$ Y. Mao $(\triangle)$

State Key Laboratory of Genetic Engineering, Institute of Genetics, School of Life Sciences, Fudan University, Shanghai, P.R. China,

200433

Tel. +86-21-65643573; Fax +86-21-65642502

e-mail: ymmao@fudan.edu.cn
(CaMKs). These kinases all phosphorylate CREB at a particular residue, serine 133 (Ser133). Phosphorylation of Ser133 is believed to promote CREB-dependent transcription by allowing CREB to interact with a transcriptional coactivator, CREB-binding protein (CBP). Previous studies have established that the domain encompassing Ser133 on CREB, known as the kinase-inducible domain (KID), interacts specifically with a short domain in the CBP termed the KIX domain and that this interaction depends on the phosphorylation of Ser133 (Karpinski et al. 1992). CREB binds to its DNA target sequence as a dimer. Dimerization occurs through a conserved structural motif at the C-terminus of the protein formed by a heptad repeat of leucine residues, referred to as the leucine zipper. DNA binding is mediated by a basic domain, a lysine- and arginine-rich stretch just amino-terminal to the leucine zipper. In addition, the mechanism by which CREB activates transcription varies depending on the stimulus. In some cases, signaling pathways target additional sites on CREB or on proteins associated with CREB, permitting CREB to regulate distinct programs of gene expression under different conditions of stimulation (Shaywitz and Greenberg 1999).

During our large-scale sequencing analysis of human cDNA libraries, we cloned a full-length cDNA of the human $C R E B 4$ gene encoding a homologue of the mouse $C R E B 3$ gene. The nucleotide sequence has been submitted to the Genbank/EMBL Database with accession number AF468007. The cDNA consists of $1592 \mathrm{bp}$ and contains an open reading frame (ORF) of $1188 \mathrm{bp}$ encoding a protein of 395 amino acids. A basic region leucine zipper (BRLZ) is located at residues 215-279 of the protein sequence according to a domain analysis performed by using the web service of the U.S. National Center for Biotechnology Information (NCBI; RPS-BLAST) The cDNA is considered to be full length because there is an upstream frame-stop codon (TGA) and a polyA signal (AATAAA) after the ORF. A bioinformatics analysis performed by using BLASTp revealed that CREB4 shared a high degree of homology (62\% identity and $72 \%$ similarity) with mouse CREB3. Various levels of homology with hCREB3 and hCREB-H were also detected (Fig. 1). 
hCREB 4 MCREB3 hCREB3 hCREB $-\mathrm{H}$
--MDLGIPDLLDAWLE-PPEDIFSTGSVIELGLHCPPLEVPVTRLQEQGLQGWKSGGDRG : - MELGCPELL----E-PEEDIESTGSFLELGFNGPASKVPVTR--------

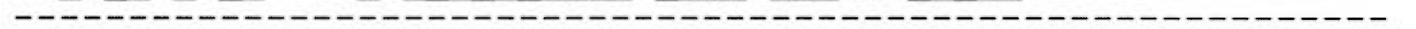
MNTDLAAGKMASAACSMDEIDSEELLDLLFDRQDGILRHVELGE--------GWGHVKDQ:
57

hCREB4 : CGLQESEPEDELKLFIDPNEVYCSE---ASPGSDSGISED-P-CHPDSPPAPRATSSPMI : 112 MCREB3 : -GLQKSEPDDELNLEIDPNMIHCSE---TSPGRDSGVSED-E-----GSPAQQASSSPAL : 87 hCREB3 : - 3 :- 16 hCREB-H : QVLPNPDSDDELSSILGSGDSLPSSPLWSPEGSDSGISEDLESDPQDTPPRSGPATSPAG : 112

hCREB4 : YEVVY--EAGALERMQGETGPNV---------GLISIQLDQWSPAFMV--------- : 149 MCREB3 : YEVVY--DSGTLQGTQREAGPTF---------GLISIQIDQWTPALMV--hCREB3 : LEESGDLGTAPDEAVRAPLDWALPLSEVPSDWEVDDLICSLLSPPASLNILSSSNPCLVH : 76 hCREB-H : CHPAQPGKGPCISYHPGNSCSTTTPGPVIQVPEASVTIDLEMNSPGGRICAEKPADPVDL : 172

hCREB4 : ------PDSCMVSELPFDAHAHIIPRAGTVAPVPCTTLLPCQT-TELTDEERRIIGQEG : 201 MCREB3 : -----DDACTVSGLPSDSHRHILPRVSTRAPAPPAAMPSCOHHLFLTDEEKQLIAQEG : 177 hCREB3 : HDHTYSLERETVSMDLESESCRKEGTQMTPQHMEELAEQEIAR--IVLTDEEKSLLEKEG : 134 hCREB-H : SPRCNLTVKDLLISGSSGDLQQHHLGASYLLREGAG----HCQE-IVLIEDEKKLLAKEG : 227
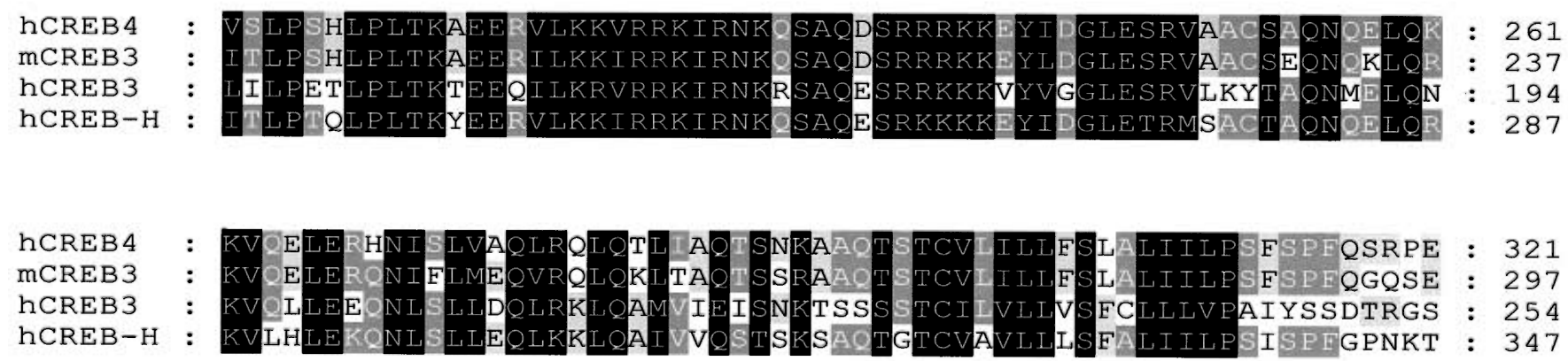

hCREB4 : AGSEDYQPHGVTSRNILTHKDVTENLETQVVES-------------MCREB3 : ARPEDYQLHCVISRNILTHENVTENLESPVLKSKLEE----------hCREB3 : LPAE---HGVLSRQLRALPSEDPYQLELPALQSEVPKDSTHQWLDGSDCVLQAPGNTSC : 310 hCREB-H : ESPGDFAPVRVFSRTLHNDAASRVAADAVPGSEAPGPRPEADTTREESPGSPGADWGEQD: 407

hCREB4 : - $4----=--=-=-=----$ RLREPPGAK- -DANGSTRTLLEKMGGKPRPSGRTRSVLHA : 392 mCREB3 : - $3----------==-=-=-------$ LPEAPTTNGSTKTHLKMRVKARPPGQIRGMVHT: 367 hCREB3 : LLHYMPQAPSAEPPLEWPEPDLESEPLCRGPILPLQANLTRKGGWLPTGSPSVILQDRYS: 370 hCREB-H : TANLTNSTEELDNATLVLRNATEGLGQVALLDWVAPGPSTGSGRAGLEAAGDEI=----- : 461

hCREB4 : DEM : 395
MCREB3 : DEM : 370
hCREB3 : G-- : 371
hCREB-H : $=-=:$

Fig. 1. Alignment of human cAMP response element-binding (CREB) protein CREB4 with mCREB3, hCREB3, and hCREB-H. Numbers on the right refer to the last amino acid in each corresponding line. Identity is indicated by a black box, and similarity is indicated by a gray box 
Table 1. Nucleotide sequence of exon-intron junctions (bold) of the human CREB4 gene

\begin{tabular}{|c|c|c|c|c|c|}
\hline 3' Splice acceptor & Exon & Size (bp) & 5' Splice donor & Intron & Size (bp) \\
\hline cDNAend GGGGTTTGGCTG & 1 & 112 & TCTTCAGAACAGgtaatgcttggg & 1 & 211 \\
\hline gccttcctgcagAAGCATGGATCT & 2 & 178 & GACCGTGGCTGTgtgagtgtgacg & 2 & 230 \\
\hline ttcctactgtagGGCCTTCAAGAG & 3 & 251 & CCAGCTAGGTCA gtgttctttgtg & 3 & 157 \\
\hline actccagatcagTGGAGCCCAGCA & 4 & 118 & TGTACAACCCTGgtgagtcttggt & 4 & 3062 \\
\hline ccctaccccagCTGCCCTGTCAA & 5 & 93 & CСССТCACCAAGgtaacatgcttc & 5 & 135 \\
\hline tgggcctccaagGCAGAGGAGAGG & 6 & 107 & GCTGGAGAGCAGgtacgcctgggt & 6 & 116 \\
\hline cctacattctagGGTGGCAGCCTG & 7 & 69 & GAGGCACAACATgtgagtgaaagc & 7 & 116 \\
\hline ctcctttcccagCTCCTTGGTAGC & 8 & 85 & ACTTGTGTTTTGgtaccattagtc & 8 & 155 \\
\hline gettcctcccagATTCТТСТТТTТ & 9 & 97 & AGCCTCACGGAGgtgagaggcaag & 9 & 155 \\
\hline ttcacctcacagTGACTTCCAGAA & 10 & 481 & ATAAACGTTTTA & & \\
\hline
\end{tabular}

Intron sequences are shown in lowercase and exon sequences in uppercase

Fig. 2. Tissue distribution of $C R E B 4$ expression

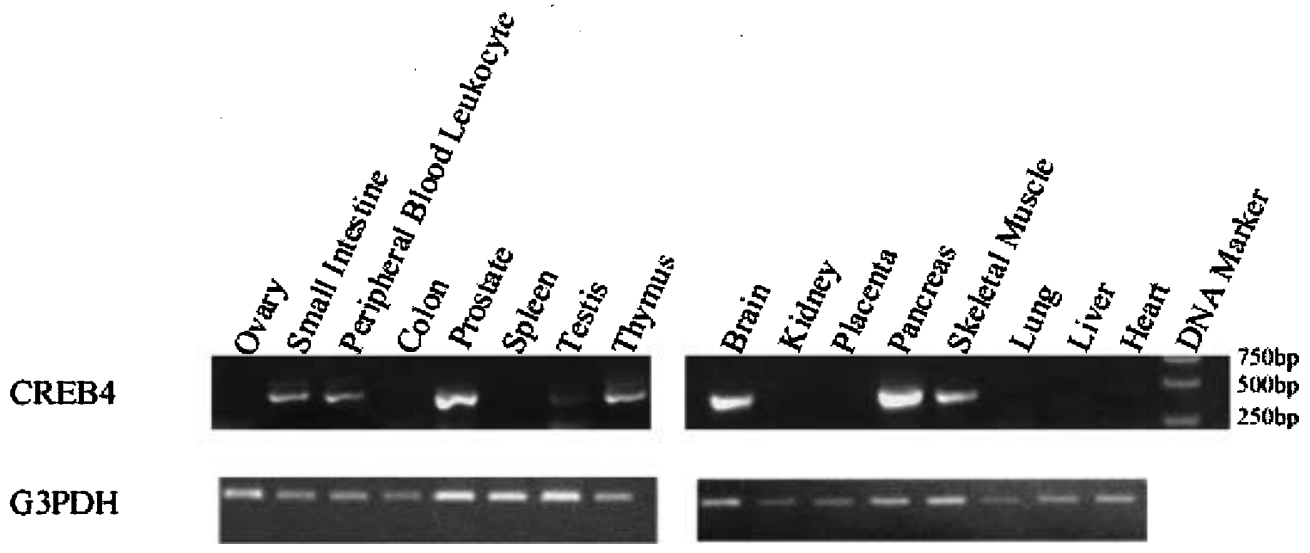

To determine the chromosomal localization of the human $C R E B 4$ gene, the international human genome database on the NCBI web site was used. The gene was mapped to contig NT-021933, spanning 5928bp and located at 1q21.3. A comparison of our cDNA with the genome sequence of $C R E B 4$ suggested that the gene consisted of ten exons and nine introns. All sequences at the exonintron junctions were consistent with the AG-GT rule (Table 1). Other genes located nearby were those for B-cell CLL/lymphoma 9 (1q21), S100 calcium-binding protein A2 (1q21), and CD5 antigen-like protein (scavenger receptor cysteine-rich family)(1q21-q23).

The tissue distribution of CREB4 expression was determined by multipletissue cDNA (MTC) panel polymerase chain reaction (PCR) analysis. Two human MTC panels (Clontech, Palo Alto, CA, USA) were used as the PCR template, in accordance with the manufacturer's protocol. The sequences for the human $C R E B 4$-specific primer pair were 5' -tgccagcttcagtccattccagag-3' (CREB4 F, from 1046$1070 \mathrm{bp}$ ) and 5'-caaatgcagactaggcctcaggcag-3' (CREB4 R, from $1454-1478 \mathrm{bp}$ ). Thirty-five cycles of amplification (30 s at $94^{\circ} \mathrm{C}, 1 \mathrm{~min}$ at $65^{\circ} \mathrm{C}$, and $1 \mathrm{~min}$ at $72^{\circ} \mathrm{C}$ ) were performed using ELONGASE DNA polymerase (GIBCO BRL, Carlsbad, CA, USA). The PCR product of CREB4 was then resolved on a $1.5 \%$ Metaphor agarose gel (FMC, Philadelphia, PA, USA). In total, 16 human tissues were tested, including brain, heart, lung, liver, kidney, skeletal muscle, placenta, spleen, thymus, prostate, testis, ovary, small intestine, colon, and leukocyte (Fig. 2). Our data revealed a transcript in prostate, brain, pancreas, and skeletal muscle, and a lower level of expression was detected in small intestine, testis, leukocyte, and thymus, whereas in heart, lung, liver, kidney, placenta, spleen, ovary, and colon, specific bands of the transcript could not be detected.

Earlier studies have shown that the cAMP-response element-binding protein (CREB) is a mammalian transcription activator that binds as a dimer to a broad spectrum of inducible promoters. CREB activity is modulated by several signaling agents (protein kinase $\mathrm{A}, \mathrm{Ca}^{2+}$, and transforming growth factor beta) and via functional interactions with cell-specific transcription factors. In addition, CREB can activate transcription constitutively and repress the activity of several other transcriptional activators (HausSeuffert and Meisterernst 2000).

Recently, it was suggested that CREB plays a pivotal role in dopamine (DA) receptor-mediated nuclear signaling and neuroplasticity (Andersson et al. 2001). Interestingly and importantly, some data suggested that phosphorylated CREB could recruit p53 tumor suppressor protein, modify its transcriptional activity, and increase the expression of p21 (Waf1/Cip1), a p53-regulated cyclin-dependent kinase inhibitor (Arnould et al. 2002).

We report here a novel human $C R E B$ gene (CREB4), which is located at chromosome 1q21.3 according to a bioinformatics analysis. CREB4 is $1592 \mathrm{bp}$ long and encodes a protein of 395 amino acid residues in which a basic region 
leucine zipper domain was identified. By means of a Blastx search at NCBI, we found that the predicted protein shared high homology with mouse CREB3 (identity $62 \%$, similarity $72 \%$ ). The expression pattern of the human CREB4 gene revealed transcripts in prostate, brain, pancreas, and skeletal muscle and a lower expression level in small intestine, testis, leukocyte, and thymus, whereas in heart, lung, liver, kidney, placenta, spleen, ovary, and colon, no specific bands of the transcript could be detected. Further studies will focus on the expression of the CREB4 protein and its function.

\section{References}

Andersson M, Konradi C, Cenci MA (2001) cAMP response elementbinding protein is required for dopamine-dependent gene expression in the intact but not the dopamine-denervated striatum. J Neurosci 21:9930-9943

Arnould T, Vankoningsloo S, Renard P, Houbion A, Ninane N, Demazy C, Remacle J, Raes M (2002) CREB activation induced by mitochondrial dysfunction is a new signaling pathway that impairs cell proliferation. EMBO J 21:53-63

Haus-Seuffert P, Meisterernst M (2000) Mechanisms of transcriptional activation of cAMP-responsive element-binding protein CREB. Mol Cell Biochem 212:5-9

Karpinski BA, Morle GD, Huggenvik J, Uhler MD, Leiden JM (1992) Molecular cloning of human CREB-2: an ATF/CREB transcription factor that can negatively regulate transcription from the cAMP response element. Proc Natl Acad Sci USA 89:4820-4824

Shaywitz AJ, Greenberg ME (1999) CREB: a stimulus-induced transcription factor actived by a diverse array of extracellular signals. Annu Rev Biochem 68:821-861 